\title{
Cost effectiveness of shortening screening interval or extending age range of NHS breast screening programme: computer simulation study
}

\author{
Rob Boer, Harry de Koning, Anthony Threlfall, Peter Warmerdam, Andrew Street, Ellis Friedman,
} Ciaran Woodman

Seep 388 and
Editorial by
Werneke and
McPherson
Department of
Public Health,
Instituut
Maatschappelijke
Gezondheidszorg,
Erasmus University
Rotterdam, PO Box
1738, 3000 DR
Rotterdam,
Netherlands
Rob Boer,
informatician
Harry de Koning,
assistant professor
Peter Warmerdam,
econometrist
Centre for Cancer
Epidemiology,
University of
Manchester,
Manchester
M20 4QL
Anthony Threlfall,
research officer
Ciaran Woodman,
director
York Health
Economics
Consortium,
University of York,
York YO1 5DD
Andrew Street,
senior research fellow
Department of
Public Health
Medicine, West
Pennine Health
Authority, Oldham
OL1 2PN
Ellis Friedman,
director of public
health
Correspondence to:
Dr Boer
boer@mgz.fgg.eur.nl

BMJ 1998;317:376-9

\begin{abstract}
Objective: To compare the cost effectiveness of two possible modifications to the current UK screening programme: shortening the screening interval from three to two years and extending the age of invitation to a final screen from 64 to 69 .

Design: Computer simulation model which first simulates life histories for women in the absence of a screening programme for breast cancer and then assesses how these life histories would be changed by introducing different screening policies. The model was informed by screening and cost data from the NHS breast screening programme.

Setting: North West region of England.

Main outcome measures: Numbers of deaths prevented, life years gained, and costs.

Results: Compared with the current breast screening programme both modifications would increase the number of deaths prevented and the number of life years saved. The current screening policy costs £2522 per life year gained; extending the age range of the programme would cost $£ 2612$ and shortening the interval £2709 per life year gained. The marginal cost per life year gained of extending the age range of the screening programme is $£ 2990$ and of shortening the screening interval is $£ 3545$.

Conclusions: If the budget for the NHS breast screening programme were to allow for two more invitations per woman, substantial mortality reductions would follow from extending the age range screened or reducing the screening interval. The difference between the two policies is so small that either could be chosen.

\section{Introduction}

In 1988 the NHS breast screening programme, on the recommendation of an expert committee, began screening women aged 50-64 years every three years. However, the committee also concluded that the optimum frequency of screening and the age range likely to benefit from breast screening were still undetermined. ${ }^{1}$ We used the computer simulation package Microsimulation Screening Analysis (MISCAN) to compare the cost effectiveness of two possible modifications to the current UK screening programme: shortening the screening interval from three to two years and extending the age of invitation to a final screen from 64 to 69 .

\section{Methods}

A full description of the Microsimulation Screening Analysis model has been published. ${ }^{2}$ In brief, the model first simulates life histories for women in the absence of a screening programme for breast cancer and then assesses how these life histories would change as a consequence of introducing different screening policies.

The natural course of breast cancer is modelled as a progression from no breast cancer through preclinical cancer to clinical disease. Women reside in the first state (no breast cancer) before entering one of five preclinical states. There is an in situ state and four invasive states according to the tumour size (T state): $\leqslant 5 \mathrm{~mm}$ (T1a), >5-10 mm (T1b), >10-20 mm (T1c), and $>20 \mathrm{~mm}(\mathrm{~T} 2+)$. A cancer may be detected at screening, become clinically apparent in any one of these states, or if undiagnosed progress to the next preclinical state. The two end states of the model are death from breast cancer and death from other causes.

The model was set up using data from the Dutch screening trials at Utrecht and Nijmegen to provide estimates of the mean duration of the preclinical phase for women in different age groups and the mean duration of cancer in each of the five preclinical states. The dwelling time of a cancer in each preclinical state is assumed to follow an exponential distribution, and the rate at which cancers progress from the preclinical to the clinical state is inferred from the observed incidence and distribution of stages of clinically diagnosed cancers in the population being studied.

When modelling the performance of a screening programme, key indicators include the mean duration of the screen detectable phase, the sensitivity of the test, and the improvement in prognosis for screen detected cancers. The mean preclinical screen detectable period assumed in the model was based on data from the Dutch screening projects at Nijmegen and Utrecht and varied from 1.8 years at age 35 to 6.2 years at age 70 .

The sensitivity of the screening test is assumed in the model to be the probability of detecting a cancer in the preclinical screen detectable state. For women aged over 50 it is fixed as $0.4,0.65,0.8,0.9$, and 0.95 for in situ disease, T1a, T1b, T1c, and $\mathrm{T} 2+$ tumours respectively. The improvement in prognosis for screen detected cancers was derived from the results of the Swedish breast screening trials. ${ }^{3}$

\section{Applying model to UK population}

The North West health region has a population of 4.1 million and is covered by five NHS breast screening programmes. The largest of these, the Manchester breast screening programme, has screened over 120000 women and reported cancer detection rates similar to those elsewhere in the United Kingdom. ${ }^{4}$ The number and size of cancers detected at a first and second screen and the occurrence and size of interval cancers in this programme have been used to inform the model. Estimates of screening and diagnostic costs are based on this programme assuming that two view 
mammography is used at the first screen and single view mammography at subsequent screens. Treatment costs are derived from various sources, but primarily the Christie Hospital NHS Trust in Manchester. Full details of the costing, including sensitivity analysis, have been published. ${ }^{5}$ Both costs and effects are discounted at $6 \%$.

To simulate the life histories of women with breast cancer before a screening programme is introduced the model requires information on the age, distribution of stage, and survival of women with breast cancer. Neither the prescreening distribution of stage nor stage specific survival rates before screening was introduced were available for the North West's population. However, the prescreening stage distribution in Scotland $^{6}$ and in East Anglia (J McCann, East Anglian Cancer Registry, personal communication) was similar to that of the control population in the Utrecht screening trial. We therefore assumed that the prescreening stage distribution in the North West was similar to that used in setting up the computer model. The stage distribution in women aged 50-69 at diagnosis in the Utrecht control population was: $4.6 \%$ in situ, $1.5 \% \mathrm{~T} 1 \mathrm{a}$, $6.3 \% \mathrm{~T} 1 \mathrm{~b}, 32.6 \% \mathrm{~T} 1 \mathrm{c}$, and $55 \% \mathrm{~T} 2+$. Having assumed this stage distribution, we derived stage and age specific survival rates by fitting the North West's observed mortality for 1987 to the observed incidence for 1987. This produced an overall five year survival for women aged $50-59$ and women aged $60-69$ of $67 \%$ and $68 \%$ respectively. A life table describing the probability of dying from causes other than breast cancer in the North West was used to derive the number of life years gained per breast cancer death prevented.

The model was unable to simulate the detection rate and distribution of stages observed at first screening in the North West. More small cancers were observed in the North West than were predicted by the model. This discrepancy was resolved by assuming a longer screen detectable preclinical phase for small tumours. When it was assumed that small tumours (less than $10 \mathrm{~mm}$ ) dwelt in a screen detectable phase for twice as long as that used in the initial set up, the model adequately fitted the detection rate and stage distribution observed at first screening in the North West.

This model was used to simulate the effects and costs of three screening programmes for the North West: firstly, the current UK screening policy, in which women aged between 50 and 64 are invited for screening every three years; secondly, screening every three years but extending the age of women screened from 64 to 69 years; and, finally, reducing the screening interval from three to two years while maintaining the current age range. Attendance for screening was assumed to fall by $0.5 \%$ for each year of age, from $74.2 \%$ at age 50 to $67.9 \%$ at age 70 ; attendance at repeat invitations was assumed to be $78 \%$ higher than among those who attended the previous invitation. Each screening programme was assumed to run for 27 years.

\section{Results}

The final model adequately predicted the rates of screen detected cancers observed at the first and second screening round, the distribution of stages observed at the first screen but not the distribution observed at the second screen, and the interval cancer rates observed after a first screen (tables 1-3).
Table 1 Observed and computer modelled rates of detection of breast cancer per 1000 women screened

\begin{tabular}{lccccc} 
& \multicolumn{2}{c}{ First screening } & & \multicolumn{2}{c}{ Second screening } \\
\cline { 2 - 3 } \cline { 6 - 6 } Age & Observed & Modelled & & Observed & Modelled \\
\hline $50-54$ & 5.5 & 4.6 & & NA & 3.7 \\
\hline $55-59$ & 5.6 & 5.6 & & NA & 4.0 \\
\hline $60-64$ & 7.1 & 7.6 & & NA & 5.5 \\
\hline All ages & 6.0 & 5.9 & & 4.7 & 4.6 \\
\hline
\end{tabular}

$\mathrm{NA}=$ not available.

Table 2 Observed and computer modelled distribution of tumour stage ( $T$ state). Values are percentages of women

\begin{tabular}{lccccc} 
& \multicolumn{2}{c}{ First screening } & & \multicolumn{2}{c}{ Second screening } \\
\cline { 2 - 3 } \cline { 5 - 6 } Stage & Observed & Modelled & & Observed & Modelled \\
\hline In situ & 14.2 & 14.9 & & 17.0 & 15.1 \\
\hline $\mathrm{T} 1 \mathrm{a}$ & 8.6 & 9.1 & 3.8 & 13.0 \\
\hline $\mathrm{T} 1 \mathrm{~b}$ & 31.5 & 31.0 & & 29.6 & 38.8 \\
\hline $\mathrm{T} 1 \mathrm{c}$ & 31.0 & 30.2 & & 33.9 & 25.6 \\
\hline $\mathrm{T} 2+$ & 14.7 & 14.8 & & 15.7 & 7.5 \\
\hline
\end{tabular}

Table 3 Observed and computer modelled rates of interval cancer after first screening per 10000 women screened

\begin{tabular}{lcc} 
Months after screen & Observed & Modelled \\
\hline $0-11$ & 5.5 & 5.4 \\
\hline $12-23$ & 9.2 & 9.8 \\
\hline $24-35$ & 14.9 & 13.0 \\
\hline Total & 29.5 & 28.2 \\
\hline
\end{tabular}

Table 4 Effects and costs of three screening policies for breast cancer

\begin{tabular}{lccc} 
& $\begin{array}{c}\text { Current } \\
\text { programme }\end{array}$ & $\begin{array}{c}\text { Extension to } \\
\text { age 69 }\end{array}$ & $\begin{array}{c}\text { Two year } \\
\text { interval }\end{array}$ \\
\hline Effectiveness (no discounting) & & & \\
\hline Reduction in mortality (\%) & 12.8 & 16.4 & 15.3 \\
\hline Deaths prevented/year* & 147 & 188 & 175 \\
\hline Total deaths prevented & 4079 & 5311 & 4880 \\
\hline Life years gained & 66187 & 78221 & 81322 \\
\hline Costs (£m) (6\% discounted) & & & \\
\hline Screening & 26.8 & 34.7 & 36.9 \\
\hline Diagnosis (screening) & 17.4 & 21.1 & 19.7 \\
\hline Diagnosis (outside screening) & -11.1 & -14.1 & -12.9 \\
\hline Primary treatment & 6.2 & 9.1 & 7.1 \\
\hline Adjuvant therapy & -1.5 & -2.1 & -1.8 \\
\hline Follow up & 2.9 & 4.0 & 3.5 \\
\hline Advanced disease & -9.8 & -13.1 & -11.8 \\
\hline Total & 30.9 & 39.6 & 40.6 \\
\hline Effectiveness (6\% discounted) & & & \\
\hline Deaths prevented & 1229 & 1636 & 1457 \\
\hline Life years gained & 12251 & 15161 & 14987 \\
\hline Cost effectiveness (6\% discounted) & & & \\
\hline Cost per death prevented (£) & 25142 & 24205 & 27865 \\
\hline Cost per life year gained (£) & 2522 & 2611 & 2709 \\
\hline Marginal cost effectiveness/life \\
$\begin{array}{l}\text { year gained (£) } \\
\text { *In a steady state. }\end{array}$ & 2990 & 3545 \\
\hline
\end{tabular}

Table 4 provides a summary of the costs and effects of the three screening policies compared with no screening. This suggests that the current North West screening programme reduces mortality by $12.8 \%$, preventing 4079 deaths over 27 years; this is equivalent to 66187 life years gained or 12251 life years discounted to present values.

Screening to age 69 reduced mortality by $16.4 \%$, preventing 5311 deaths over 27 years (equivalent to 
Key messages

- Computer modelling suggested that the current breast screening programme in North West England will reduce total female breast cancer mortality by $12.8 \%$

- Extending the programme to age 69 would reduce mortality by $16.4 \%$ at a marginal cost per life year saved of $£ 2990$ while reducing the interval to two years would reduce mortality by $15.3 \%$ at a marginal cost per life year saved of $£ 3545$

- Extending the age range prevents more deaths from breast cancer but shortening the interval gains more life years

- If the budget for the NHS breast screening programme would allow for two more invitations per woman either of the two options could be chosen others, ${ }^{7}$ and the results from the model have been used to evaluate screening programmes in several European countries. ${ }^{8-11}$ For the model to simulate the detection rates and distribution of stages observed at first screening in the North West we had to assume a longer preclinical detectable phase for smaller tumours than was estimated from the Dutch pilot projects and the Dutch national screening programme. A longer preclinical detectable phase is in accordance with a lower threshold of detection of breast cancer at screening.

The model adequately simulated the number of cancers occurring in the interval between screens and those detected at a second screen, but it predicted a better stage distribution at repeat screening than was observed. This discrepancy has been reported before ${ }^{11}$ and is being investigated by the Erasmus team. It is unlikely, however, that the discrepancy substantially affected the conclusion since a better stage distribution at repeat screens is modelled in all policy options and the overall reduction in mortality predicted for each screening policy option is not greater than the reductions reported from the randomised trials of breast screening.

\section{Which is the best policy?}

In cost effectiveness analysis of programmes whose main effect is to extend life the usual measure of benefit is life years gained. Compared with the current breast screening programme both of the alternatives evaluated offer improved effectiveness; both are predicted to increase the number of life years gained and number of deaths prevented. Choice of policy will depend on which outcome measure is chosen, whether discounting is undertaken, and whether costs are considered. If health effects are not discounted and costs are ignored, extending the age range prevents more deaths but reducing the screening intervals results in more life years gained.

Whether either of the proposed changes to the programme is cost effective depends on the value the NHS is willing to place on improvements in the effectiveness of the programme. Compared with no screening and assuming a discount rate of $6 \%$ applied to all costs and outcomes the current programme costs $£ 25142$ per death prevented and £2522 per life year saved. The cost per life year saved is higher with both proposed changes, but extending screening to women aged 69 reduces the cost per death prevented. If cost per death prevented is taken as the appropriate outcome measure then extending the age of screening is more cost effective than the current policy. This policy also has a smaller increase in cost per life gained than two year screening compared with the current programme.

In conclusion, if the budget for the NHS breast screening programme would allow for two more invitations per woman, the computer model predicts that the difference between extending the age range screened or reducing the screening interval from three to two years is so small that either could be chosen. This conclusion was not affected by using upper and lower estimates of the costs of screening, diagnosis, and treatment or by varying the discount rate of costs and benefits.

We thank Luke Readman of the Manchester Breast Screening Service and Liz Twelves of the North West Regional Breast
The computer model was developed and refined at the Erasmus University, Rotterdam. It has been validated with data from the Netherlands ${ }^{2}$ and Sweden, ${ }^{3}$ assumptions underpinning the model have been evaluated by 
Screening Quality Assurance Team for providing accurate cost and screening data.

Contributors: EF initiated the project. AT and CW collated screening and epidemiological data. RB, HK, and PW did the simulation analysis. AS collated and analysed economic data. Al authors participated in consultations on study design and the interpretation of the findings. RB, AT, and CW wrote the paper with input from all members of the project team. $\mathrm{RB}$ is guarantor of the work.

Funding: NHS Breast Screening Programme on behalf of the National Advisory Committee on Breast Cancer Screening. Conflict of interest: None.

1 Forrest APM. Report to the health ministers of England, Wales, Scotland and Northern Ireland by a working group chaired by Sir Patrick Forrest. London: HMSO, 1987

2 van Oortmarssen GJ, Habbema JD, van der Maas PJ, de Koning HJ, Collette $\mathrm{HJ}$, Verbeek AL, et al. A model for breast cancer screening. Cancer 1990;66:1601-12.

3 de Koning HJ, Boer R, Warmerdam PG, Beemsterboer PM, van der Maa PJ. Quantitative interpretation of age-specific mortality reductions from the Swedish breast cancer-screening trials. J Natl Cancer Inst 1995;87:1217-23.

4 Chamberlain J, Moss SM, Kirkpatrick AE, Michell M, Johns L. NHS breast screening programme results for 1991-2 [published correction appears in BMJ 1993;307:543]. BMJ 1993;307:353-6.

5 Street AD, Posnett J, Threlfall AG, Woodman CBJ, Twelves E, Friedman EHI, et al. Economic evaluation of proposed changes to the breast screening programme. York: York Health Economics Consortium, University of York, 1996. (Report P20/03.)

6 Scottish Cancer Therapy Network. Scottish breast cancer audit 1987 and 1993. Report to the chief scientist and CRAG. Edinburgh: Scottish Cancer Therapy Network, 1996

7 Brown ML, Fintor L. Cost-effectiveness of breast cancer screening preliminary results of a systematic review of the literature. Breast Cancer Res Treat 1993;25:113-8.

8 de Koning HJ, van Ineveld BM, van Oortmarssen GJ, de Haes JC, Collette $\mathrm{HJ}$, Hendriks JH, et al. Breast cancer screening and cost-effectiveness; policy alternatives, quality of life considerations and the possible impact of uncertain factors. Int J Cancer 1991;49:531-7.

9 van Ineveld BM, van Oortmarssen GJ, de Koning HJ, Boer R, van de Maas PJ. How cost-effective is breast cancer screening in different EC countries? Eur J Cancer 1993;12:1663-8.

10 Beemsterboer PM, de Koning HJ, Warmerdam PG, Boer R, Swart F, Dierks ML, et al. Prediction of the effects and costs of breast-cancer screening in Germany. Int J Cancer 1994;58:623-8.

11 Paci E, Boer R, Zappa M, de Koning HJ, van Oortmarssen GJ, Crocetti F et al. A model-based prediction of the impact on reduction in mortality by a breast cancer screening programme in the city of Florence, Italy. Eu J Cancer 1995;3:348-53.

(Accepted 20 March 1998)

\section{Safety and toxicity of amphotericin B in glucose $5 \%$ or intralipid $20 \%$ in neutropenic patients with pneumonia or fever of unknown origin: randomised study}

Patrick Schöffski, Mathias Freund, R Wunder, D Petersen, C H Köhne, H Hecker, U Schubert, A Ganser

\begin{abstract}
Objective: To compare the feasibility of treatment, safety, and toxicity of intravenous amphotericin B deoxycholate prepared in either glucose or intralipid for empirical antimycotic treatment of neutropenic cancer patients.

Design: Single centre stratified, randomised non-blinded phase II study.

Setting: University hospital providing tertiary clinical care.

Subjects: 51 neutropenic patients (leukaemia (35), lymphoma (11), solid tumours (5)) with refractory fever of unknown origin (24) or pneumonia (27). Interventions: Amphotericin B $0.75 \mathrm{mg} / \mathrm{kg} /$ day in $250 \mathrm{ml}$ glucose $5 \%$ solution or mixed with $250 \mathrm{ml}$ intralipid $20 \%$, given on eight consecutive days then alternate days, as a 1-4 hour infusion.

Main outcome measures: Feasibility of treatment, subjective tolerance (questionnaire), and objective toxicity (common toxicity criteria of the National Cancer Institute).

Results: Study arms were balanced for age, sex, underlying malignancy, renal and liver function, and pre- and concomitant treatment with antibiotics and nephrotoxic agents. No statistically significant or clinically relevant differences were found between the treatment groups for: daily or cumulative dose and duration of treatment with amphotericin $\mathrm{B}$; incidence and time of dose modifications or infusion duration changes related to toxicity; dose or duration of symptomatic support with opiates, antipyretics, or
\end{abstract}

antihistamines; renal function; subjective tolerance; most common toxicity scores; course of infection; and incidence of treatment failures. Patients treated with amphotericin $\mathrm{B}$ in intralipid were given fewer diuretics $(\mathrm{P}<0.05)$ and therefore had more peripheral oedema $(\mathrm{P}<0.01)$ and needed less potassium supplementation $(\mathrm{P}<0.05)$ than patients given amphotericin in glucose. Acute respiratory events were more common in the intralipid arm $(\mathrm{P}<0.05)$. Conclusions: Amphotericin B $0.75 \mathrm{mg} / \mathrm{kg} /$ day in intralipid given on eight consecutive days then alternate days provides no benefit and is associated with potential pulmonary side effects possibly because of fat overload or an incompatibility of the two drugs.

\section{Introduction}

Amphotericin B is regarded as the agent of choice for treatment of life threatening mycoses in neutropenic patients because of its broad antimycotic activity. ${ }^{1}$ It is conventionally given intravenously in glucose $5 \%$, as a colloidal suspension with the detergent sodium deoxycholate. Amphotericin B is associated with a high incidence of renal toxicity, potassium loss, fever, and chills. Attempts have been made to overcome its dose limiting renal toxicity. ${ }^{2}$ Well tolerated, highly expensive liposomal formulations are commercially available, and can be used in patients developing renal toxicity after exposure to amphotericin B deoxycholate.

Non-liposomal lipid emulsions are also known to reduce toxic effects of amphotericin in vitro and in vivo and have been given to patients. $^{3-11}$ Intralipid, a
Department of Haematology/Oncology, Hanover University Medical School, D-30625 Hanover, Germany

Patrick Schöffski, physician

R Wunder,

physician

U Schubert,

documentation

assistant

A Ganser, professor of medicine

Department of Clinical Chemistry D Petersen, physician

Biometrical Department

H Hecker, professor of biometric. Department of Haematology/Oncology, Rostock University, D-18055 Rostock, Germany

Mathias Freund, professor of medicine C H Köhne, physician

Correspondence to: Dr Schöffski Patrick_Schoffski@ compuserve.com

BMJ 1998;317:379-84 